\title{
Ende der ärztlichen Dominanz in der Psychiatrie
}

\author{
The End of Physicians' Dominance in Psychiatry
}

\section{Bibliografie}

DOI http://dx.doi.org/

10.1055/s-0030-1248478

Psychiat Prax 2010; 37:

216-218

(c) Georg Thieme Verlag KG

Stuttgart · New York .

ISSN 0303-4259

\section{Korrespondenzadressen}

\section{Prof. Dr. Stefan Priebe}

Queen Mary University

of London

Academic Unit, Newham Centre for Mental Health

London E13 8SP, UK

s.priebe@qmul.ac.uk

\section{Prof. Dr. Markus Weih}

Universitätsklinikum Erlangen

Psychiatrische und Psychotherapeutische Klinik

Schwabachanlage 6

91054 Erlangen

markus.weih@uk-erlangen.de

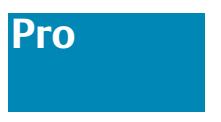

Die moderne Psychiatrie wurde im 19. Jahrhundert von Ärzten als eigenständiges Fach in der Medizin etabliert. Ärzte waren es auch, die die Psychiatrie in der Folgezeit weiterentwickelten und in der 2. Hälfte des 20. Jahrhunderts grundlegend reformierten. Sie waren bei diesen Initiativen nicht allein, aber haben sie doch dominiert. Auch heute sind die meisten Professoren in der Psychiatrie Ärzte, und Ärzte haben mehr rechtliche Kompetenzen und Verantwortung in der psychiatrischen Versorgung als jede andere Berufsgruppe. Auf Psychiaterkongressen kann man weiterhin den Eindruck gewinnen, die ärztliche Dominanz in der Psychiatrie sei ungebrochen. Aber ist das wirklich so?

Man kann die Psychiatrie zumindest in 3 Bereichen dominieren: 1.) in der „Macht“, psychiatrische Versorgung zu bestimmen und psychiatrische Einrichtungen zu leiten; 2.) in der psychiatrischen Wissenschaft und 3.) in der therapeutischen Kompetenz. Betrachten wir jeden Aspekt einzeln.

Im täglichen Alltag erleben viele von uns, dass Politiker, Manager und Kassenangestellte längst die administrative Macht in der psychiatrischen Versorgung übernommen haben. Zwar gibt es in vielen Einrichtungen ärztliche Leiter oder gar Ärzte als Geschäftsführer. Deren Befugnis ist aber viel eingeschränkter als etwa bei Anstaltsleitern früherer Zeiten, z. B. von Gremien und Verwaltungschefs. Zudem bleibt offen, ob ärztliche Leiter überhaupt noch als Ärzte zu zählen sind, da sie häufig selbst bereits zu reinen Managern geworden sind und ihre ärztliche Identität im Sinne der täglichen Patientenversorgung in der Vergangenheit liegt.

Kommen wir zur Wissenschaft, deren Regeln sich in den letzten 30 Jahren grundlegend geändert haben. Als Folge eines zunehmenden, globalisierten Wettkampfs um Forschungsgelder und Impactfaktoren ist ein viel höherer Grad an Experti- se und Spezialisierung erforderlich, um sich durchsetzen zu können. Zudem erweist sich Grundlagenforschung als besser publizierbar als angewandte klinische Forschung. Für die Psychiatrie, wie auch für andere Fächer in der Medizin, hat das 2 Konsequenzen: Zum einen sind Wissenschaftler mit Ausbildungen in Grundlagenfächern viel besser qualifiziert, innovative Konzepte und Methoden zu entwickeln und die Wissenschaft voranzutreiben. Zum anderen haben diejenigen Psychiater, die wirklich noch als Ärzte arbeiten, also täglich Patienten sehen und behandeln, praktisch kaum noch eine Chance, mit den Spezialisten mitzuhalten, die sich den ganzen Tag nur auf die Forschung konzentrieren. Das hat dazu geführt, dass selbst in psychiatrischen Zeitschriften die wissenschaftlich bedeutsamen Arbeiten zunehmend von Biologen, Psychologen, Epidemiologen und anderen Berufsgruppen, aber eben nicht mehr von Psychiatern führend publiziert werden.

Der 3. Aspekt, die klinische Kompetenz, ist wahrscheinlich am schwierigsten und sicherlich kontrovers. Die Kompetenz, die für Patienten relevant ist, liegt nicht in theoretischem Wissen um Ursachen, komplexe Zusammenhänge und diagnostische Feinheiten ohne therapeutische Konsequenzen. Was Patienten, und auch die Öffentlichkeit, primär interessiert, ist, ob wir ihnen helfen können, d.h. unsere konkreten therapeutischen Möglichkeiten. Aus meiner Sicht haben Psychiater sich hinsichtlich ihrer eigenen therapeutischen Fähigkeiten in unglückseliger Weise auf die Verschreibung von Medikamenten fokussiert und dabei selbst über die Zeit entqualifiziert. Was für diese Entwicklung entscheidend war, ist schwer zu klären, z.B. das Bestreben, anderen Medizinern gleich zu sein, die unsäglichen finanziellen $\mathrm{Zu-}$ wendungen der Pharmaindustrie, die nicht nur Individuen korrumpieren, sondern auch Inhalte von Fortbildungen und Kongressen beeinflussen, oder schlicht eine defensive Abgrenzung der eige- 
nen Rolle. Die genauen Gründe sind in diesem Zusammenhang auch unerheblich. Es bleibt, dass die Ärzte in der Psychiatrie den Zug verpasst haben, andere Kompetenzen voranzutreiben und zu ihren eigenen zu machen. Möglicherweise wäre dieses Manko weniger bedeutsam, wenn die Pharmakotherapie sich so fortentwickelt hätte, wie das vor 30 Jahren wohl viele gehofft hatten. Tatsächlich sind in dieser Zeit aber trotz einer größeren Auswahl kaum neue Medikamente auf den Markt gekommen, die der wissenschaftlichen Evidenz zufolge in ihrer therapeutischen Effektivität den damals zur Verfügung stehenden deutlich überlegen wären. Kaum eine andere spezialisierte Berufgruppe kann sich leisten, ihre Kompetenz auf einen Bereich mit so wenig Fortschritt aufzubauen.

Manch ein Leser mag hier protestieren und einwerfen, dass Psychiater ja auch eine psychotherapeutische Ausbildung sowie Kompetenzen in der Gesprächsführung und im Regeln des sozialen Umfeldes von Patienten haben. Stimmt, oft jedenfalls. In psychologischen Behandlungsverfahren sind viele klinische Psychologen aber schlichtweg viel besser ausgebildet (von anderen Aspekten wie neuropsychologischen Testverfahren im Übrigen gar nicht zu reden). Und in der Kommunikation mit Patienten und sozialen Interventionen mögen viele Ärzte sehr erfahren sein, systematisch ausgebildet sind sie nicht.

Und schließlich haben die Ärzte ihre frühere Dominanz auch im Verhältnis mit ihren Patienten - sowie z.T. auch deren Angehörigen - verloren. Das Arzt-Patienten-Verhältnis ist häufiger ein partnerschaftliches denn ein paternalistisches, und eine zunehmende Zahl von internetinformierten Patienten sucht den Ratschlag, aber nicht die Anordnung des Arztes.

Ärzte sind in der Psychiatrie sicher nicht bedeutungslos geworden. Als einzelne Berufsgruppe sind sie wahrscheinlich immer noch wichtiger als jede andere, obwohl Pflegekräfte wegen ihrer größeren Zahl und Psychologen wegen ihrer spezifischen Kompetenz anderer Meinung sein mögen. Dominieren Ärzte allein aber die gesamte Psychiatrie? Die Antwort ist wohl eher nein. Ob wir uns das wünschen oder für die Psychiatrie für günstig halten, ist eine andere Frage. Die zunehmende Bedeutung anderer Berufsgruppen kann für die Psychiatrie insgesamt und für ihre Patienten Vorteile haben, für Psychiater sollte es in jedem Fall ein Anlass sein, die eigene Rolle und Kompetenz zu überdenken. Die Dominanz in der Versorgungsgestaltung und Leitung von Einrichtungen ist sicher nicht zurückzuerobern. Eine beratende Funktion als Experte und eine Stimme innerhalb einer multiprofessionellen Gruppe sind vielleicht keine schlechte Alternative. In der Wissenschaft können Ärzte angesichts des zunehmenden Drucks zur Spezialisierung auch nicht mehr dominieren. Was sie aber beeinflussen können, ist ihre klinische Kompetenz. Sie können Aus-, Fort- und Weiterbildungen verändern und mit neuen Inhalten füllen. Ohne die Pharmakotherapie zu vergessen, können psychotherapeutische Ansätze, soziale Interventionen und die spezifische therapeutische Kommunikation mit psychisch kranken Patienten (auch und insbesondere außerhalb formaler Psychotherapie) stärker berücksichtigt werden [1]. Und darüber hinaus könnten Psychiater auch richtig und systematisch dafür ausgebildet werden, mit anderen Berufsgruppen und Patienten in einer nicht-dominanten Rolle optimal zusammenzuarbeiten und dabei eine zentrale integrative Funktion übernehmen.

\section{Kontra}

„Ein großer Teil der Psychiatrie wie sie heute gelehrt wird, verlangt keine medizinische Ausbildung. Wie Freud betonte, kann eine Psychotherapie auch von nichtmedizinischen Spezialisten durchgeführt werden. Warum sollte man dann an eine medizinische Fakultät gehen? Je stärker die Biologie das Wesen der Psychiatrie zu verändern beginnt, desto größer wird vermutlich die Anzahl begabter Medizinstudenten, die von der Psychiatrie angezogen werden“ [2].

Die Psychiatrie ist ärztlich dominiert. Im Gegensatz zu anderen Disziplinen gibt es aber einen engeren Kontakt zu angrenzenden Geisteswissenschaften. Die Erfolge der Psychotherapie und die Grenzen der Psychopharmakotherapie haben die ärztliche Dominanz in der Psychiatrie infrage gestellt. Diese Frage ist berechtigt und verdient es, adäquat diskutiert zu werden.

\section{Ökonomie}

Ärzte sind Generalisten („Allgemeinmediziner der Psyche“), Psychologen Spezialisten.

Psychiater können die meisten organischen Krankheiten diagnostizieren oder benachbarte Fachgebiete zurate ziehen. Rechtlich hat der Patient im Krankenhaus das Recht, unter Aufsicht eines Facharztes behandelt zu werden. In der Psych-PV, welche derzeit den Personalbedarf bestimmt, ist unter $\S 7$ festgelegt, dass eine Psychiatrie unter „ärztlicher Leitung“ betrieben wird und auch in der Außendarstellung stellt für Patienten eine ärztliche Leitung ein Gütesiegel einer Klinik dar, so wie ein Dr. med. auch heute noch ein Gütesiegel für eine gute Behandlungsqualität ist. 2013 wird die Psychiatrie in Deutschland auf ein pauschalierendes leistungsorientiertes Vergütungssystem umgestellt werden. Basis sind Codierrichtlinien nach denen die „Verantwortung für die Dokumentation und Codierung und Diagnosen und Prozeduren beim behandelnden Arzt liegt“" [3].

Analog zur somatischen Medizin, wo z.B. der Chirurg die Appendizitis diagnostiziert und auch die Appendektomie codiert, wird dies künftig auch für die Psychiatrie gelten. Konkret bedeutet dies, dass Ärzte für die Diagnosestellung und Therapie psychischer Erkrankungen verantwortlich sind, ansonsten können die Leistungen nicht vergütet werden. Im OPS-Code 1-903 steht beispielsweise, dass die „Diagnose und Therapien durch ein multiprofessionelles Team unter Leitung eines Facharztes“ durchgeführt werden.

Psychiater müssen verschiedenste Therapiesituationen betreuen können, von der nächtlichen Krisenintervention nach einem Suizidversuch über eine differenzierte gerontologische Psychopharmakotherapie bis hin zur elektiven Gesprächspsychotherapie. Alle diese Leistungen müssen künftig dokumentiert werden und tragen zur Finanzierung des psychiatrischen Krankenhauses bei. Außer diesen eher rechtlichen Rahmenbedingungen ist dieses Vorgehen auch ökonomisch, da es die Therapie aus einer Hand bietet und hilft, die durch Subspezialisierungen unvermeidbare Doppeltherapie zu vermeiden.

\section{Wissenschaft}

Wissenschaftlich ist die Pharmakotherapie der Psychotherapie nicht unterlegen. Reduktionistisch betrachtet wirken Psychopharmaka auf die synaptische Übertragung ein. Die Psychotherapie hingegen wirkt - vereinfacht - über eine veränderte Genexpression auf die Effektivität der Synapsen ein.

Aus der funktionellen Perspektive wirkt die Psychotherapie über eine veränderte Aktivität bewusstseins- und sinnesnaher kortikaler Strukturen auf stimmungs- und wahrnehmungsmodulie- 
rende subkortikale Strukturen wie Hippokampus, Amygdala usw. ein.

Die Pharmakotherapie wirkt umgekehrt: Serotonin, Noradrenalin, Dopamin und Acetylcholin haben alle ihre Kerngebiete im Mittelhirn bzw. im basalen Vorderhirn und kortikale Projektionen. Unter diesem Modell betrachtet wird es einfacher zu verstehen, dass die Pharmakotherapie und die Psychotherapie gleich wirksam sind bzw. sich ergänzen.

Dies könnte eine Erklärung dafür sein, dass es kaum Studien gibt, die eine eindeutige Überlegenheit der Psychotherapien zeigen. Eher schein es so zu sein, dass die Psychopharmakotherapie überlegen ist (z.B. bei Depressionen [4]).

Im Gegensatz dazu zeigte sich in Studien immer wieder, dass eine Kombination aus Medikation und Psychotherapie die wirksamste Behandlung z.B. für Depressionen darstellt [5] oder bei Pharmakoresistenz eine zusätzliche Psychotherapie keinen zusätzlichen Nutzen bringt [6].

Die meisten Psychotherapiestudien bei Depressionen wurden für leichte und mittelschwere Störungen durchgeführt. Spätestens bei einer schweren Depression mit oder ohne Suizidalität oder psychotischen Symptomen wird die Notwendigkeit einer medizinischen stationären Aufnahme mit Psychopharmakotherapie kaum infrage gestellt. Wenn sich dann Psychotherapie und Pharmakotherapie biochemisch-funktionell und klinisch gleichwertig sind bzw. sich ergänzen, muss sich dann auch gefragt werden, warum für die Psychotherapie ein Spezialist erforderlich ist, wenn ein Arzt beide Therapien „aus einer Hand“ bündeln kann. Die chronische Finanzknappheit im Gesundheitswesen darf nicht dazu führen, vermeintlich leichter verfügbare und preiswerteren psychologischen Psychotherapeuten die Therapien zu überlassen, wo die wissenschaftliche Evidenz fehlt und es zu einer Zerfaserung der Therapien kommen würde.

\section{Nachwuchsförderung}

In der ärztlichen Approbationsordnung gibt es das Fach Psychiatrie und Psychotherapie; in der Weiterbildungsordnung den entsprechenden Facharzt. Ein beginnender Mediziner hat also ein klares Berufsziel vor Augen. Für psychologische Psychotherapeuten, handelt es sich um eine kostspielige Weiterbildung nach einem abgeschlossenen Studium. Ein klares Berufsziel hin zur kli- nischen Psychiatrie kann ein Bachelorstudent der Psychologie nicht erkennen und muss zunächst ein praxisfernes geisteswissenschaftliches, verschultes Studium durchlaufen. Dies erklärt, warum Psychologen später dann oft Schwierigkeiten haben sich im „System Krankenhaus“ zurechtzufinden durch die fehlende praktische Ausbildung, fehlende biologische Grundlagen und unterschiedliche Denkweisen und Terminologien.

Wie im einleitenden Zitat von Kandel erwähnt, kann eine Psychotherapie auch ohne medizinische Fachkenntnisse erfolgreich betrieben werden. Liegt jedoch kein profundes anatomisch-physiologisches Verständnis des Gehirns und seiner Funktionsweise vor, kann zwar ein therapeutischer Fortschritt erzielt werden, jedoch nicht auf der Ebene der Pathophysiologie.

Die Psychiatrie würde sich ohne Ärzte ihres eigenen wissenschaftlichen Nachwuchses berauben, die biologische Psychiatrie als ihre Grundlagendisziplin aufgeben und daneben auch noch ihre humanistischen Wurzeln vergessen. Gerade diesen Grund der Förderung des wissenschaftlichen Nachwuchses in der Psychiatrie als beste Investition in die Zukunft halten wir für den bedeutsamsten Grund für die Fortsetzung der ärztlichen Dominanz in der Psychiatrie.

\section{Literatur}

1 Priebe S, McCabe R. Therapeutic relationships in psychiatry: The basis of therapy or therapy in itself? International Review of Psychiatry 2008; 20: 521-526

2 Kandel E. A new intellectual framework for psychiatry. Am J Psychiatry 1998; 155: 457-469

3 Deutsche Kodierrichtlinien für die Psychiatrie und Psychosomatik. 2010

4 Cuijpers P, van Straten A, van Oppen P et al. Are psychological and pharmacologic interventions equally effective in the treatment of adult depressive disorders? A meta-analysis of comparative studies. J Clin Psychiatry 2008; 69: 1675-1685; quiz 1839-1641 Doi: ej07m04112 [pii]

5 Keller M, McCullough J, Klein D et al. A comparison of nefazodone, the cognitive behavioral-analysis system of psychotherapy, and their combination for the treatment of chronic depression. N Engl J Med 2000; 342: $1462-1470$

6 Kocsis J, Gelenberg A, Rothbaum B et al. Cognitive behavioral analysis system of psychotherapy and brief supportive psychotherapy for augmentation of antidepressant nonresponse in chronic depression: the REVAMP Trial. Arch Gen Psychiatry 2009; 66: 1178-1188 Doi: 66/11/ 1178 [pii]\&\#xD;\&\#xA;10.1001/archgenpsychiatry.2009.144 